\title{
Efficient tomography of a quantum many-body system
}

\author{
B. P. Lanyon ${ }^{\dagger},{ }^{1,2, \text { * }}$ C. Maier, ${ }^{1,2,{ }^{\dagger}}$ M. Holzäpfel, ${ }^{3}$ T. Baumgratz, ${ }^{3,4,5}$ \\ C. Hempel, ${ }^{2,6}$ P. Jurcevic, ${ }^{1,2}$ I. Dhand, ${ }^{3}$ A. S. Buyskikh, ${ }^{7}$ A. J. \\ Daley, ${ }^{7}$ M. Cramer, ${ }^{3,8}$ M. B. Plenio, ${ }^{3}$ R. Blatt,,${ }^{1,2}$ and C. F. Roos ${ }^{1,2}$ \\ ${ }^{1}$ Institut für Quantenoptik und Quanteninformation, \\ Österreichische Akademie der Wissenschaften, \\ Technikerstr. 21A, 6020 Innsbruck, Austria \\ ${ }^{2}$ Institut für Experimentalphysik, Universität Innsbruck, \\ Technikerstr. 25, 6020 Innsbruck, Austria \\ ${ }^{3}$ Institut für Theoretische Physik and IQST, \\ Albert-Einstein-Allee 11, Universität Ulm, 89069 Ulm, Germany \\ ${ }^{4}$ Clarendon Laboratory, Department of Physics, \\ University of Oxford, Oxford OX1 3PU, United Kingdom \\ ${ }^{5}$ Department of Physics, University of Warwick, Coventry CV4 7AL, United Kingdom \\ ${ }^{6}$ ARC Centre for Engineered Quantum Systems, \\ School of Physics, The University of Sydney, \\ Sydney, New South Wales 2006, Australia. \\ ${ }^{7}$ Department of Physics and SUPA, University of Strathclyde, Glasgow G4 ONG, UK \\ ${ }^{8}$ Institut für Theoretische Physik, Leibniz Universität Hannover, Hannover, Germany
} (Dated: July 7, 2017)

*Electronic address: ben.lanyon@uibk.ac.at

${ }^{\dagger}$ These authors contributed equally to this work. 
Quantum state tomography (QST) is the gold standard technique for obtaining an estimate for the state of small quantum systems in the laboratory [1]. Its application to systems with more than a few constituents (e.g. particles) soon becomes impractical as the effort required grows exponentially with the number of constituents. Developing more efficient techniques is particularly pressing as precisely-controllable quantum systems that are well beyond the reach of QST are emerging in laboratories. Motivated by this, there is a considerable ongoing effort to develop new state characterisation tools for quantum many-body systems [2-11]. Here we demonstrate Matrix Product State (MPS) tomography [2], which is theoretically proven to allow the states of a broad class of quantum systems to be accurately estimated with an effort that increases efficiently with constituent number. We use the technique to reconstruct the dynamical state of a trapped-ion quantum simulator comprising up to 14 entangled and individually-controlled spins (qubits): a size far beyond the practical limits of QST. Our results reveal the dynamical growth of entanglement and description complexity as correlations spread out during a quench: a necessary condition for future beyond-classical performance. MPS tomography should therefore find widespread use to study large quantum many-body systems and to benchmark and verify quantum simulators and computers.

An MPS is a way of expressing a many-particle wave function which, for a broad class of physical states, offers a compact and accurate description with a number of parameters that increases only polynomially (i.e. efficiently) in system components. [12]. MPS tomography recognises that the information required to identify the compact MPS is typically accessible locally; that is, by making measurements only on subsets of particles that lie in the same neighbourhood [2]. In such cases, the total effort required to obtain a reliable estimate for the state in the laboratory increases at most polynomially in system components [2, 6]. States suited to MPS tomography and its generalisations to higher dimensions [2] include those with a maximum distance over which significant quantum correlations exist between constituents (locally-correlated states) e.g. the $2 \mathrm{D}$ cluster states - universal resource states for quantum computing — and the ground states of a broad class of 1D systems [13-15]. We find that MPS tomography is also well-suited to characterise the states generated during the dynamical evolution of systems with short-ranged interactions, as found in many physical systems (Methods and [16]).

Consider an $\mathrm{N}$-component quantum system initially in a product state (or other locally- 
correlated state) in which interactions are abruptly turned on. In the presence of finite-range interactions, information and correlations spread out in the system with a strict maximum group velocity [17--19]. Therefore, after a finite evolution time, there is a maximum distance over which correlations extend in the system (the correlation length, L), beyond which correlations decay exponentially in distance. The information required to describe the state is largely contained in the local reductions: the reduced states (density matrices) of all groups of neighbouring particles contained within $L$. In 1D systems, such states can be described by a compact MPS [15, 20] and, to identify and certify the total $N$-component MPS, the experimentalist need only perform the measurements required to reconstruct the local reductions [16]. Each local reduction can be determined by full QST, requiring measurements in at most $3^{L}$ bases. Since the number of local reductions increases only linearly in $N$, the total number of measurement bases scales efficiently in this parameter. The local reduction estimates are passed to a classical algorithm which finds an MPS estimate in a time polynomial in $N$ [2, 7] (see Figure 1). We find that the total number of measurements required to obtain a desired fidelity for the state reconstruction also scales efficiently in $N$ (Methods).

Our strategy is not restricted to $1 \mathrm{D}$ systems nor to those with strictly finite range interactions. In any of those cases, we benefit from the fact that MPS tomography and its natural generalisations to higher spatial dimensions [2] make no prior assumptions about the form of the state in the laboratory (e.g. that it is pure or well-described by a compact MPS), because the state estimate can be certified: an efficient assumption-free lower bound on the fidelity with the laboratory state $\rho_{\text {lab }}$ is provided [2]. For example, the correlation length $L$ need not be known a priori. If, after measurements on k-sites, the certified minimum fidelity $F_{c}^{k}$ between the MPS estimate $\left|\psi_{c}^{k}\right\rangle$ and the state in the laboratory $\rho_{\text {lab }}$ is deemed not high enough, then one can try again for larger $k$. Generalisation of our method to higher spatial dimensions and to mixed-state estimates using matrix product operators [6, 7] is possible, although no general certification method is currently known for mixed states [29].

For finite range interactions, the correlation length $L$ can increase at most linearly in time as entanglement grows and spreads out in the system, demanding exponentially growing number of measurements to estimate each local reduction [21, 22]. This puts practical limits on the evolution time until which the system state can be efficiently characterised: once correlations have spread out over the whole system the effort for MPS tomography becomes the same as full QST. MPS tomography is able to verify evolution towards classically-intractable regimes: as the system evolves, the 
size of the local reductions required to obtain an accurate pure MPS description should continue to increase (as seen in our data).

Our quantum simulator consists of a string of trapped ${ }^{40} \mathrm{Ca}^{+}$ions. In each ion $j=1 \ldots N$, two electronic states encode a spin-1/2 particle. Under the influence of laser-induced forces, the spin interactions are well described by an ' $\mathrm{XY}$ ' model in a dominant transverse field $B$, with Hamiltonian $H_{X Y}=\hbar \sum_{i<j} J_{i j}\left(\sigma_{i}^{+} \sigma_{j}^{-}+\sigma_{i}^{-} \sigma_{j}^{+}\right)+\hbar B \sum_{j} \sigma_{j}^{z}$. Here $J_{i j}$ is an $N \times N$ spin-spin coupling matrix, $\sigma_{i}^{+}\left(\sigma_{i}^{-}\right)$is the spin raising (lowering) operator for spin $i$ and $\sigma_{j}^{z}$ is the Pauli $Z$ matrix for spin $j$. Interactions reduce approximately with a power-law $J_{i j} \propto 1 /|i-j|^{\alpha}$ with distance $|i-j|$. Here $1.1<\alpha<1.6$, for which the predominant feature of spreading wave packets of correlations is nevertheless evident [23-25]. Applying MPS tomography to study complex out-of-equilibrium states, generated by interactions that are not strictly finite-range, represents a most stringent test of its scope of application.

The largest application of full QST was for a simple 8 qubit W-state, employing measurements in 6561 different bases taken over ten hours [26]. We begin experiments with 8 spin (qubit) quench dynamics, and accurately reconstruct complex 8-spin entangled states using measurements in 27 bases taken over ten minutes. We measure in sufficient bases to reconstruct all $k$-local reductions of individual spins $(k=1)$, neighbouring spin pairs $(k=2)$ and spin triplets $(k=3)$, during simulator evolution starting from the initial highly-excited Néel state $|\phi(0)\rangle=|\uparrow, \downarrow, \uparrow, \downarrow \ldots\rangle$ (Methods). The local measurements directly reveal important properties: single-site 'magnetisation' shows how spin excitations disperse and then partially refocus (Figure 2a); in the first few milliseconds, strong entanglement develops in all neighbouring spin pairs and triplets, then later reducing, first in pairs then in triplets, consistent with correlations spreading out over more spins in the system (Figure $2 \mathrm{c}-\mathrm{d})$.

Certified fidelity lower bounds $F_{c}^{k} \leq\left\langle\psi_{c}^{k}\left|\rho_{\text {lab }}\right| \psi_{c}^{k}\right\rangle$ from MPS tomography during the 8-spin quench are shown in Fig 3a. The results closely match an idealised model where MPS tomography is applied to exact local reductions of the ideal time-evolved states $|\phi(t)\rangle$ (Methods). Measurements on $k=1$ sites at $t=0$ yield a certified MPS state reconstruction $\left|\psi_{c}^{1}\right\rangle$, with $F_{c}^{1}=0.98 \pm 0.01$ and $\left|\left\langle\psi_{c}^{1} \mid \phi(0)\right\rangle\right|^{2}=0.98$, proving that the system is initially well described by a pure product Néel state. As expected, the fidelity lower bounds based on single-site measurements rapidly degrade as the simulator evolves, falling to 0 by $t=2 \mathrm{~ms}$. Nevertheless, an accurate MPS (pure-state) description is still achieved by measuring on larger $(k=2)$ and larger $(k=3)$ reduced sites. The model fidelity bounds $F_{c}^{3}$ begin to drop after $t=2 \mathrm{~ms}$, consistent with the time at which the information 
wavefronts are expected to reach next-nearest-neighbours (light-like cones, Figure 2a), allowing for correlations beyond 3 sites to develop. Measurements on $k=3$ sites reveal an MPS description with more than 0.8 fidelity up to $t=3 \mathrm{~ms}$, before the lower bound rapidly drops to 0 at $6 \mathrm{~ms}$. This is consistent with the model and the entanglement properties measured directly in the local reductions (Figure 2b-c): At $t=3 \mathrm{~ms}$, entanglement in spin triplets maximises before reducing to almost zero at $6 \mathrm{~ms}$, as correlations have then spread out to include more distant spins. Beyond $t=3 \mathrm{~ms}$ it becomes increasingly difficult to uniquely distinguish (and certify) the global state based on 3-site local reductions (although the estimate can still be a good description).

The data in Figure 3a clearly reveal the generation and spreading-out of entanglement during simulator evolution, up to 3-4 ms, and are consistent with this behaviour continuing beyond this time. To confirm this, it would be necessary to measure on increasingly large numbers of sites, demanding measurements that grow exponentially in $k$. That the amount of entanglement in the simulator is growing in time can be seen from the inset in figure 3a: the half-chain entropies of the certified MPSs $\left|\psi_{c}^{3}\right\rangle$ are seen to grow as expected for a sudden quench [27]. For all times at which $F_{c}^{3}>0$ (except $t=0$ ), the pure MPS-reconstructed states $\left|\psi_{c}^{3}\right\rangle$ are non-separable across all partitions.

Figure 3b-c compares spin-spin correlations ('correlation matrices') present in $\left|\psi_{c}^{3}\right\rangle$ at $t=3 \mathrm{~ms}$ $\left(F_{c}^{3}>0.84 \pm 0.05\right)$, with those obtained directly in the lab via additional measurements. The certified MPS captures the strong pairwise correlations in the simulator state and correctly predicts the sign and spatial profile of correlations beyond next-nearest neighbour: that is, of state properties beyond those measured to construct it (beyond $k=3$ ).

Figure 4 presents results from a 14 spin quench: far beyond the practical limits of full QST. Full QST on 14 spins would require measuring in more than 4 million bases. We reconstruct a certified MPS estimate using only 27 local measurement bases. At $t_{14}=4 \mathrm{~ms}$, strong entanglement, in neighbouring pairs and triplets, has developed right across the system. Measurements on 3 sites, at $t_{14}$ yield an MPS estimate $\left|\psi_{c}^{3}\right\rangle$ with a certified minimum fidelity of $F_{c}^{3}=0.39 \pm 0.08$. Since $F_{c}^{k}$ are only lower bounds, it is natural to ask exactly what the state fidelity is. Using the estimated 14spin MPS state $\left|\psi_{c}^{3}\right\rangle$ from MPS tomography, we perform Direct Fidelity Estimation (DFE) [4, 5] with the experimentally generated state using an additional set of measurements, obtaining a result of $0.74 \pm 0.05$ (Methods and FIG. S10).

Clearly MPS tomography provided an accurate estimate of the 14-spin simulator state, and the fidelity lower bound of $F_{c}^{3}=0.39 \pm 0.08$ is correct. However, the bound is conservative and 
even lies quite far from the one expected from an idealised system model (0.78). We find that the certification process is compromised by errors in initial state preparation, introducing mixture at the single spin level (Methods). The current increase in state initialisation error per-spin, as our current simulator is scaled-up in size, limits the ability to accurately and efficiently characterise its state. A new optical setup yielding improved single-qubit operations should allow for a small constant error-per-spin beyond 20 spins. 
Acknowledgments. Work in Innsbruck was supported by the Austrian Science Fund (FWF) under the grant number P25354-N20, by the European Commission via the integrated project SIQS, by the Institut für Quanteninformation $\mathrm{GmbH}$ and by the U.S. Army Research Office through grant W911NF-14-1-0103. All statements of fact, opinion or conclusions contained herein are those of the authors and should not be construed as representing the official views or policies of ARO, the ODNI, or the U.S. Government. We thank H. Shen and T. Brydges for experimental support in the final stage of the experiment. Work in Ulm was supported by an Alexander von Humboldt Professorship, the ERC Synergy grant BioQ, the EU projects QUCHIP and EQUAM, the US-Army Research Office Grant No. W91-1NF-14-1-0133 and the BMBF Verbundproject QuOReP. Numerical computations have been supported by the state of Baden-Württemberg through bwHPC and the German Research Foundation (DFG) through grant no INST 40/467-1 FUGG. I.D. acknowledges support from the Alexander von Humboldt Foundation. M.H. acknowledges contributions from Daniel Suess to jointly developed code used for data analysis. Work at Strathclyde is supported by the European Union Horizon 2020 collaborative project QuProCS (grant agreement 641277), and by AFOSR grant FA9550-121-0057. M.C. acknowledges: the ERC grant QFTCMPS and SIQS, the cluster of excellence EXC201 Quantum Engineering and Space-Time Research, and the DFG SFB 1227 (DQ-mat). T. B. acknowledges: EPSRC (EP/K04057X/2) and the UK National Quantum Technologies Programme (EP/M01326X/1). B. P. L. acknowledges support by the START prize of the Austrian FWF project Y 849-N20.

Author contributions. B.P.L, C.F.R, M.B.P. and M.C. developed and supervised the project; C.M., C.H., B.P.L., P.J., R.B. and C.F.R. performed and contributed to the experiments; B.P.L., M.H., T.B., C.M, C.F.R., I.D., A.B. and A.D. performed data analysis and modelling; B.P.L. wrote the manuscript, with contributions from all authors. 


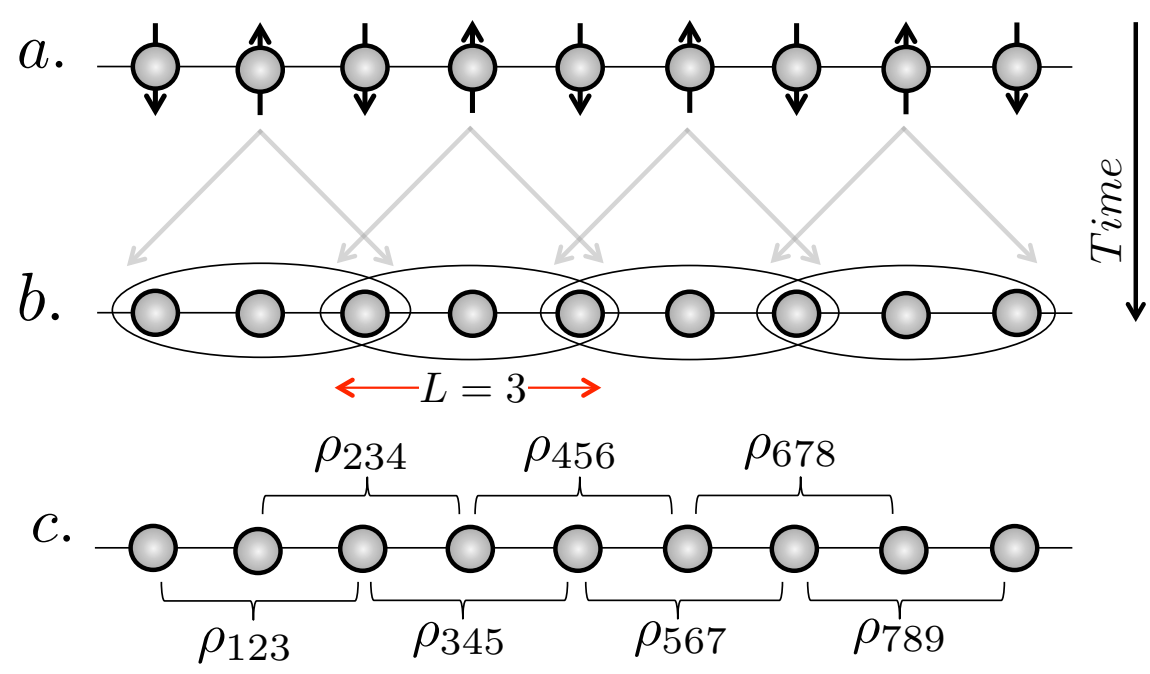

FIG. 1: Generation and efficient characterisation of locally-correlated quantum states. a. Quantum spins fixed on a 1D lattice and initialised into some separable pure state. Finite-range spin-spin interactions are then abruptly turned on. In the subsequent dynamics, quantum correlations spread out with a maximum group velocity [17,-19, 23, 28], producing light-like cones (grey arrows, only a few are shown) and a locallycorrelated entangled state. b. After the particular evolution time shown, quantum correlations have spread to neighbouring spin triplets (not all shown). The established correlation length is $L=3$. The total $N$-spin state can be accurately described by a compact MPS, efficient in N. The correlation length increases at most linearly in time. c. To obtain an accurate MPS estimate for the state in the laboratory, the experimentalist need only perform sufficient measurements to reconstruct all $N-L+1$ neighbouring spin triplet reduced density matrices (local reductions). At any given evolution time, the experimental effort therefore increases linearly in spin number $N$. 


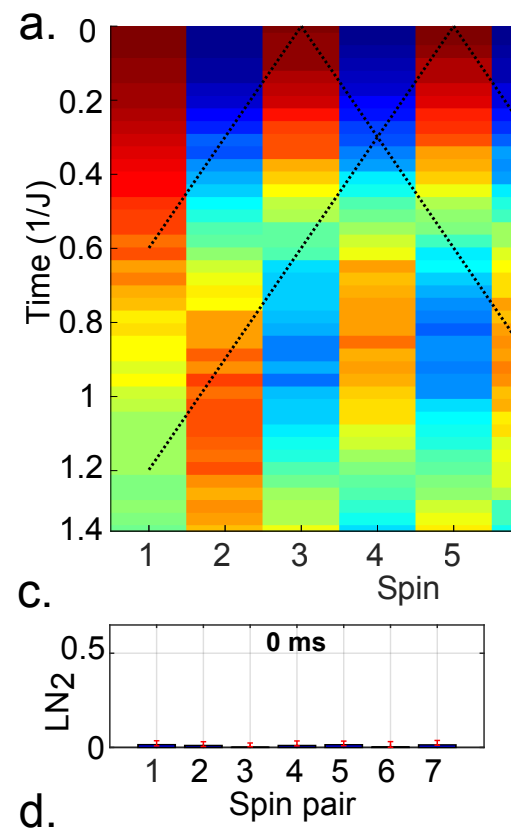

d.
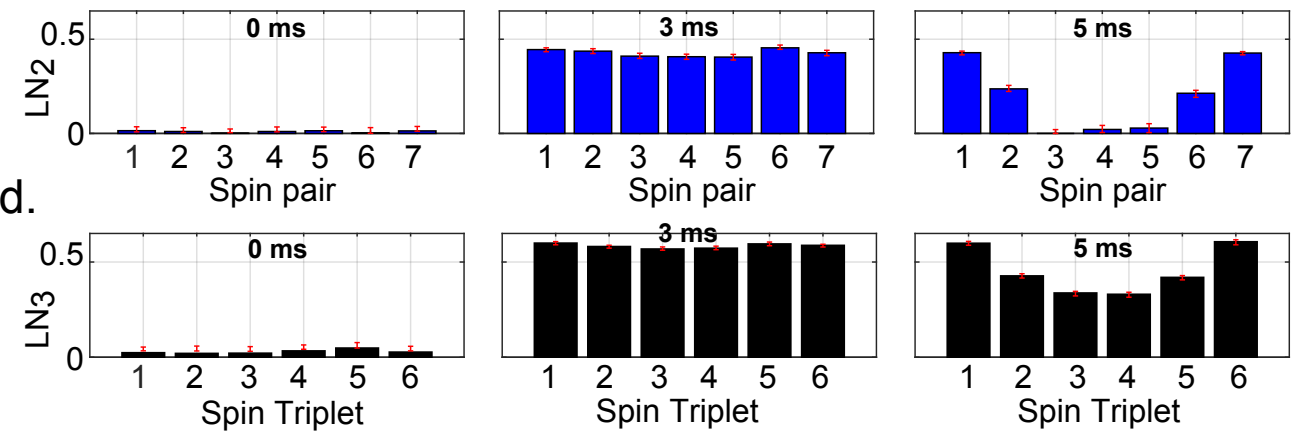

FIG. 2: Local measurement results for an 8 spin system quench. a. Single spin magnetisation $\left\langle\sigma_{i}^{z}(t)\right\rangle$ during quench dynamics. Two (out of eight) example light-like cones are shown, exemplifying an estimate for the maximum speed at which correlations spread. The interaction range $\alpha \approx 1$.6. Lefthand time axis is renormalised by the average nearest-neighbour $J$ couplings. See Methods and FIG. S4. b. Absolute value of the density matrix (local reduction) of spins $3 \& 4$ at time $t=3 \mathrm{~ms}$, reconstructed via full QST and standard maximum likelihood estimation. The state is entangled, with a bipartite logarithmic negativity of $L N_{2}=0.41 \pm 0.01$ and a fidelity with an ideal theoretical model of $0.99 \pm 0.01$. More properties of the local reductions are presented in FIG. S8. c.-d. Entanglement in all neighbouring spin pairs (c.) and spin triplets (d.) at three evolution times, as labelled: values calculated from experimentally-reconstructed density matrices, e.g. panel b. (FIG. S6). Entanglement is characterised by bipartite logarithmic negativity $\left(L N_{2}\right)$ for spin pairs and tripartite logarithmic negativity $\left(L N_{3}\right)$ for spin triplets (Methods). Error bars are 1 standard deviation derived from Montecarlo simulation of finite measurement number. 

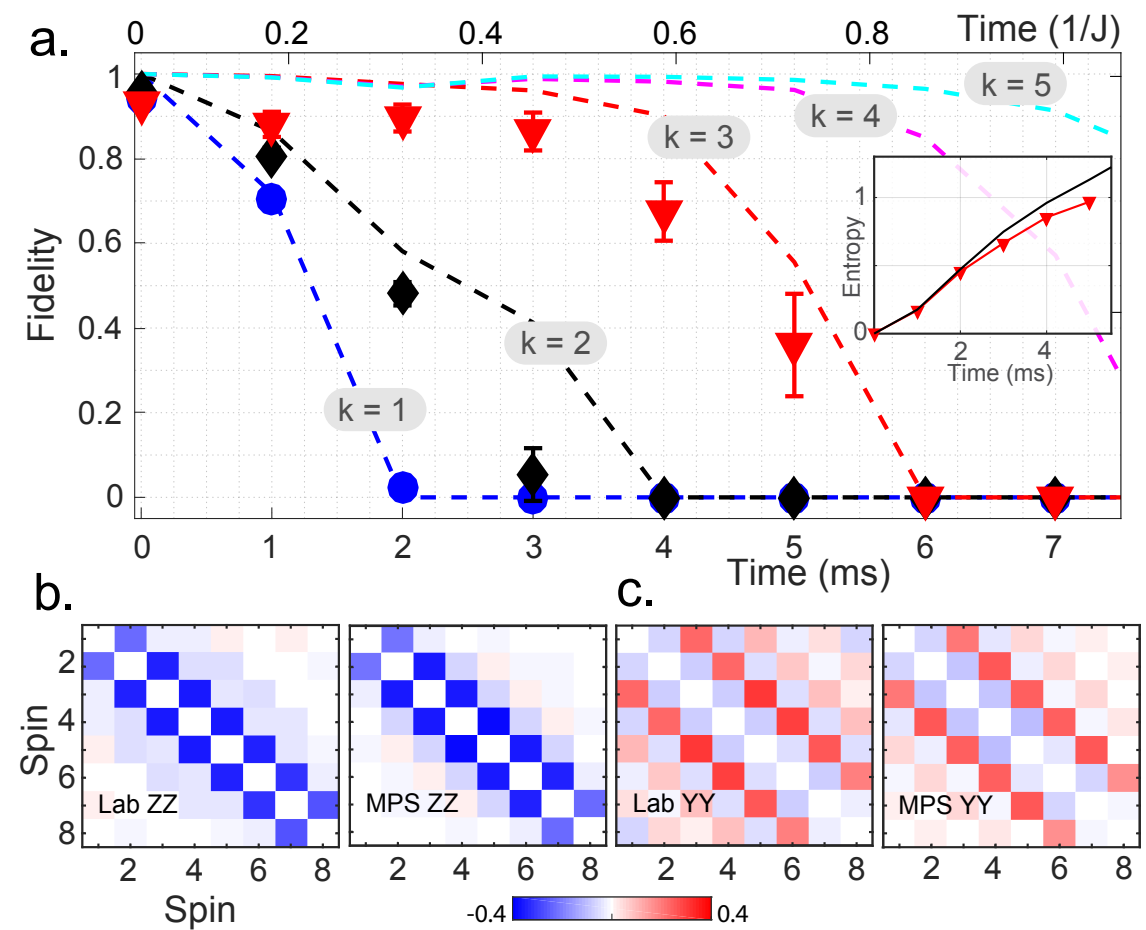

FIG. 3: MPS tomography results for an 8 spin quench. a. Certified lower bounds $F_{c}^{k}$ on the fidelity between the 8 spin MPS estimate $\left|\psi_{c}^{k}\right\rangle$, reconstructed from measurements over $k$ sites, and the quantum simulator state $\rho_{\mathrm{lab}}$, as a function of simulator evolution time. Shapes: data points with errors of 1 standard deviation confidence (uncertainty due to finite measurement number). Dashed lines: model, MPS tomography algorithms applied to exact knowledge of $k$-site local reductions derived from idealised simulator states $|\phi(t)\rangle$ (Methods). Differences between model and data are largely due to the finite number of measurements used to estimate local reductions (FIG. S2). Insert: half-chain Von Neumann entropy of the pure global state. Red triangles: from data $\left(\left|\psi_{c}^{3}\right\rangle\right)$. Black line: directly from ideal states $(|\phi(t)\rangle)$. b. Complete spin pair correlation matrices showing $\left\langle\mathrm{Z}(\mathrm{t})_{i} \mathrm{Z}(\mathrm{t})_{j}\right\rangle-\left\langle\mathrm{Z}(\mathrm{t})_{i}\right\rangle\left\langle\mathrm{Z}(\mathrm{t})_{j}\right\rangle$ at $t=3 \mathrm{~ms}$, for spins $i$ and $j$. The second term in this correlation function removes pure state classical correlations such as those present in the initial state (at $t=0$ the matrices contain all zero entries to within error). Results directly measured on $\rho_{\text {lab }}$ are compared with those present in $\left|\psi_{c}^{3}\right\rangle$ (see labels). c. Same as b. but for $\left\langle\mathrm{Y}(\mathrm{t})_{i} \mathrm{Y}(\mathrm{t})_{j}\right\rangle-\left\langle\mathrm{Y}(\mathrm{t})_{i}\right\rangle\left\langle\mathrm{Y}(\mathrm{t})_{j}\right\rangle$. Correlation matrices from an idealised model $|\phi(t)\rangle$ are presented in FIG. S7(a). Note: X,Y and Z are the standard Pauli spin operators. 


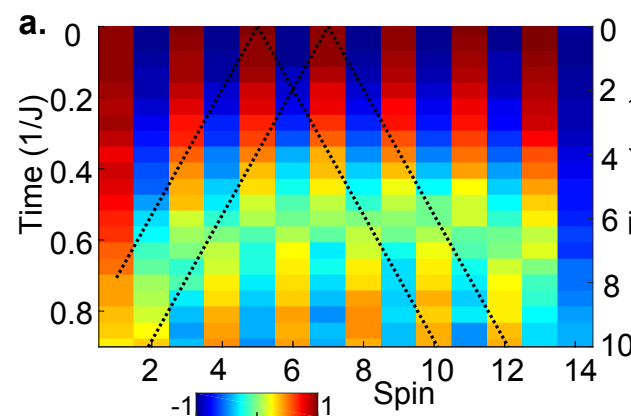

c.

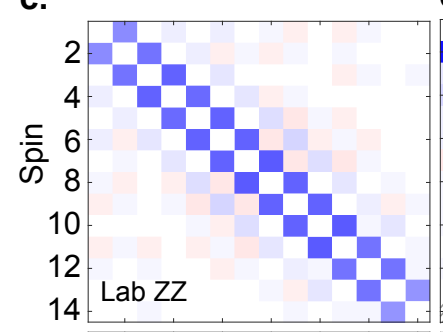

d.

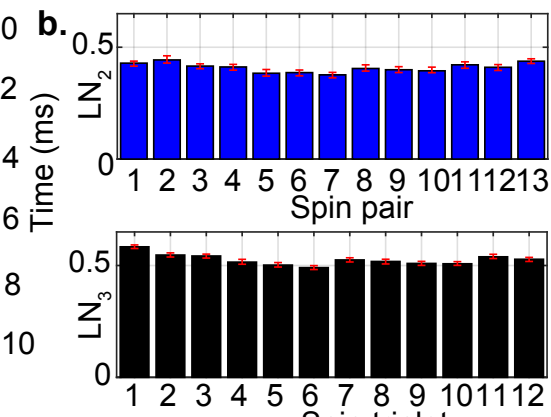

e.

Spin triplet
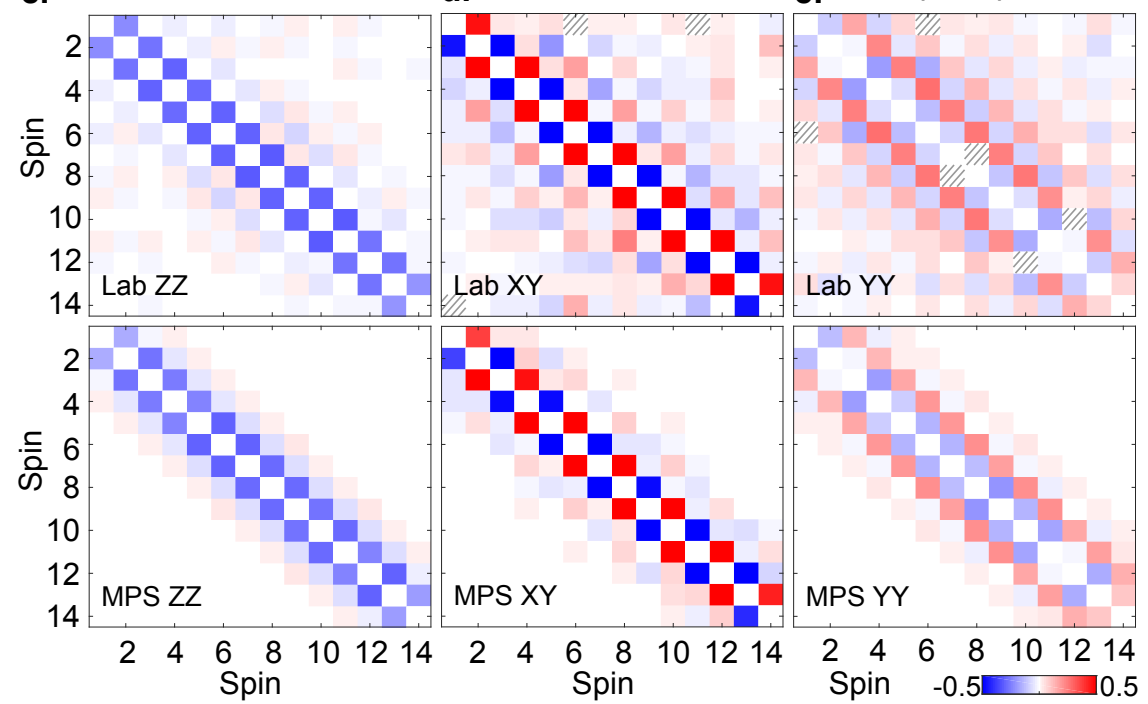

FIG. 4: MPS tomography results for a 14 spin quench. a.-b. Direct results from local measurements. a. Single spin magnetisation $\left\langle\sigma_{i}^{z}(t)\right\rangle$ with two (out of 14) example approximate light-like cones (Methods, FIG. S5). b. Entanglement in all neighbouring spin pairs (bipartite logarithmic negativity, top blue bars) and spin triplets (tripartite logarithmic negativity, bottom black bars) at $t_{14}=4 \mathrm{~ms}$ (Methods). Values calculated from experimentally-reconstructed local density matrices. Errors are 1 standard deviation derived from Montecarlo simulation of finite measurement number. c.-e. Comparison of two-spin correlation matrices directly measured in the laboratory and from the MPS estimate $\left|\psi_{c}^{3}\right\rangle$ at $t_{14}$, showing $\left\langle\mathrm{A}(\mathrm{t})_{i} \mathrm{~B}(\mathrm{t})_{j}\right\rangle-\left\langle\mathrm{A}(\mathrm{t})_{i}\right\rangle\left\langle\mathrm{B}(\mathrm{t})_{j}\right\rangle$. A, B as labelled. Not all correlations were measured in the lab (hatched squares). The 14-spin MPS estimate $\left|\psi_{c}^{3}\right\rangle$ at $t_{14}$ is seen to capture many of the correlations between spins up to 4 sites apart (see FIG. S9 for ideal model). The weak correlations over greater distances in the laboratory state develop effectively instantly in quench dynamics, due to the long-range components of our interactions. The entanglement content and distribution in $\left|\psi_{c}^{3}\right\rangle$ is consistent with the amount expected from an ideal model and the state has no separable partitions. Note: $\mathrm{X}, \mathrm{Y}$ and $\mathrm{Z}$ are the standard Pauli spin operators. 


\section{METHODS}

Trapped ion simulator and laser control. We refer to the 'axial' direction along the ion string principle axis as $z$ and the two 'radial' directions, orthogonal to the string principle axis, as the $x$ and $y$ axes. The frequencies of the centre of mass vibrational modes are: $\omega_{z}=2 \pi \times 0.214 \mathrm{MHz}$, $\omega_{y}=2 \pi \times 2.69 \mathrm{MHz}, \omega_{x}=2 \pi \times 2.71 \mathrm{MHz}$. Two electronic Zeeman states $\left|S_{1 / 2}, m=+1 / 2\right\rangle$ and $\left|D_{5 / 2}, m^{\prime}=+5 / 2\right\rangle$ in each trapped ${ }^{40} \mathrm{Ca}^{+}$ion encode the $|\downarrow\rangle$ and $|\uparrow\rangle$ states of a spin-1/2 particle, respectively, and are coupled by an electric quadrupole transition at $729 \mathrm{~nm}$. The quantum states are coherently manipulated using a Ti:Sa CW laser with a linewidth of about $1 \mathrm{~Hz}$. Two laser beam paths are employed. First, a global beam illuminates the ion string approximately equally (intensity difference between outermost and middle ion $\sim 15 \%$ ), from a direction perpendicular to the ion string $z$ axis and at an angle approximately half way between $x$ and $y$. Consider the standard Pauli spin operators $\sigma_{x}, \sigma_{y}$ and $\sigma_{z}$. The global beam is used (i) to implement standard frequency-resolved sideband cooling and optical pumping on the quadrupole transition, (ii) to perform $\sigma_{x}$ and $\sigma_{y}$ rotations simultaneously on all spins and (iii) to realise the spin-spin interaction Hamiltonian (see later). Second, a single-ion-focused beam comes in parallel to the global beam but from the opposite direction. The direction of this beam can be switched to have its focus pointing at different ions within $12 \mu \mathrm{s}$, using an acousto-optic deflector. The single-ion-focused beam is frequency-detuned by about $80 \mathrm{MHz}$ from the spin transition and thereby performs an AC-Stark rotation $\left(\sigma_{z}\right)$ on the ion in focus. The combination of the global resonant and the focused detuned beam enables arbitrary single-spin rotations [30].

Simulator initialisation. Each experimental sequence begins with Doppler cooling ( $\sim 3 \mathrm{~ms})$ and optical pumping ( $\sim 500 \mu \mathrm{s}$ ) to initialize all $N$ ions in the string into the $|\downarrow\rangle$ state. Next, all $2 N$ radial motional modes, transverse to the string, are cooled to the ground state via $\sim 10$ frequency-resolved sideband cooling pulses (10 ms in total), followed by a second frequency-resolved optical pumping step of $\sim 500 \mu \mathrm{s}$. From this pure electronic and motional quantum state $|\downarrow\rangle^{\otimes N}=|\downarrow, \downarrow, \downarrow \ldots\rangle$, the Néel state $|\uparrow, \downarrow, \uparrow \ldots\rangle$ is created by flipping every second spin to the $|\uparrow\rangle$ state. This is done using a composite pulse sequence that is robust to inhomogeneities in the laser-ion coupling strength across the string (e.g. due to weak gaussian intensity profiles). See Supp. Mat. Sec. I.B. for details.

Implementing and modelling spin-spin interactions. Spin-spin interactions, parametrised 
by $J_{i j}$, are realised via the global laser beam when containing three frequencies (a trichromatic beam), two of which off-resonantly drive all $2 N$ radial vibrational modes of the string and are symmetrically detuned by $\pm \Delta$ from the spin flip transition. The magnitude of the detuning $|\Delta|$ is larger than the highest radial COM mode $\omega_{x}$ by $2 \pi \cdot 79 \mathrm{kHz}$ ( 8 spins) or $2 \pi \cdot 76 \mathrm{kHz}$ (14 spins). The third frequency is $1 \mathrm{MHz}$ detuned from the spin flip transition and compensates for AC Stark shifts (shifts in the transition frequencies of spins across the string). When uncompensated, these AC Stark shifts are inhomogeneous across the string and arise from the Gaussian intensity profile of the global beam coupling to far off-resonant dipole transitions. Finally, an additional overall detuning $\delta$ of all three frequencies by $B=2 \pi \cdot 3 \mathrm{kHz}$ ( 8 spins) or $B=2 \pi \cdot 5 \mathrm{kHz}$ (14 spins) generates an effective transverse field (quantified by $\mathrm{B}$ in $H_{X Y}$ ).

The model spin-spin coupling matrix $J_{i j}$ is calculated from the aforementioned ion string vibrational mode frequencies, laser detunings, ionic mass and laser-ion coupling strength (see supplementary material of [23]). Maximum values are $J_{i j}=2 \pi \cdot 25 \mathrm{~Hz}\left(8\right.$ spins) and $J_{i j}=2 \pi \cdot 15 \mathrm{~Hz}(14$ spins). For more details on the experimental implementation of the spin-spin interaction Hamiltonian see [23, 24].

The full Hamiltonian is given by $H_{\text {Ising }}=\hbar \sum_{i<j} J_{i j} \sigma_{i}^{x} \sigma_{j}^{x}+\hbar \sum_{i=1}^{N}\left(B+B_{i}\right) \sigma_{i}^{z}$. That is, an Isingtype model with an overall transverse field $B$, set experimentally by the detuning $\delta$. $H$ includes small spin-dependent perturbations $B_{i}$ (at the few $\mathrm{Hz}$ level), resulting from e.g. electric quadrupole shifts which vary along the string and magnetic field curvature across the string (in addition to our standard constant 4 Gauss field). In the limit $B \gg\left|J_{i j}\right|$, which holds in all our experiments, the $H_{\text {Ising }}$ is equivalent to an $\mathrm{XY}$ model in a transverse field $\left(H_{X Y}\right)$, as given in the main text.

The ideal time-evolved states can easily be calculated by brute force matrix exponentiation for 8 spins, e.g. $|\phi(t)\rangle=\exp \left(-i H_{\text {Ising }} t / \hbar\right)|\phi(0)\rangle$. For 14 spins we use two different methods. First, as a diagnostic tool to model the $k$-spin local reductions in the laboratory, we employ the more time-efficient Krylov subspace projection methods (Arnoldi and Lanczos processes) which, in the case of sparse Hamiltonians, give a substantial speed up and well controlled error bounds [31]. Second, to calculate the full $N$-spin time evolved ideal states, and compare them with the MPS estimates, we use the library function scipy.sparse.linalg.expm_multiply [32]. Both methods produce equivalent states at the time scales considered in our experiments.

Regarding the model Hamiltonian $H_{X Y}$, all spins down $\left|\downarrow_{z}\right\rangle^{\otimes N}$ is the ground state, spins pointing up $\left|\uparrow_{z}\right\rangle$ are quasiparticle excitations which disperse and scatter in the system [24]. The Néel state is chosen for the initial state as it is highly excited ( $N / 2$ excitations) and leads to the emergence of 
locally-correlated entangled states involving all $N$ particles. Furthermore, the subsequent quench dynamics evolves in a subspace whose size, contrary to those of low-excitation subspaces (the subject of our previous work [23]), grows exponentially with $N$.

Measurements. After preparing the initial simulator state, spin interactions are abruptly turned on (a quench) and then off after any desired evolution time $t$, freezing the generated state and allowing for spin measurement. At the end of every such experimental run, the state of each spin is determined individually via the standard electron shelving technique: laser light at $397 \mathrm{~nm}$ (and $866 \mathrm{~nm}$, repumping) is sent to the ion string for several milliseconds, coupling to the strong dipole $S_{1 / 2} \rightarrow P_{1 / 2}$ transition. $397 \mathrm{~nm}$ light is scattered if the electron is in the $|\downarrow\rangle$ state and detected using a single-ion resolving CCD camera. Such a measurement setting corresponds to measuring each spin in the Z-basis, that is, projecting each spin into either of the two eigenstates of the Pauli $\sigma_{z}$ operator. Through repeated preparation and measurement, the probabilities for single-spin outcomes, or multi-spin outcomes (correlations between spin outcomes), can be estimated. We can measure any individual spin in any single-spin basis by first implementing laser-driven spin rotations that map the eigenstates of the desired single-spin-operators onto the eigenstates of the Pauli $\sigma_{z}$ operator, then carrying out electron shelving as before. In this way we could e.g. measure the first spin in the $Z$ basis, the second in $X$, the third in $Y$, etc.

MPS tomography requires the estimation of the local reduced density matrices of all blocks of $k$ neighbouring spins. For a 1D chain of $N$ spins, there are $N-k+1$ such blocks. We estimate each neighbouring $k$-spin block by measuring in $3^{k}$ bases, corresponding to all combinations of projecting each spin into the eigenstates of the three Pauli operators. Each of the $3^{k}$ settings has $2^{k}$ distinguishable outcomes, which in total adds to $3^{k} \times 2^{k}=6^{k}$ outcome probabilities. This set of $6^{k}$ local outcome probabilities is sufficient to estimate the local $k$-spin reduced density matrix. Rather than measuring the $N-k+1$ blocks separately, we implement a straightforward scheme to measure them at the same time, requiring a total of $3^{k}$ measurements bases for the entire string (see Supplementary Material Sec. III). We carry out each of the $3^{k}$ measurement settings 1000 times. The measurement outcomes are then (i) passed to the MPS tomography algorithm which finds an $N$-spin state estimate and certificate (see later) (ii) used directly to obtain local $k$-spin reduced density matrices $\rho^{(k)}$ (e.g. Figure 2 b) via standard maximum likelihood estimation [33].

Bipartite and tripartite negativity. The entanglement values in local reductions presented in 
Figures $2 \mathrm{c}-\mathrm{d}$ and $4 \mathrm{~b}$ are derived from the experimental reconstructed local states $\rho^{(k)}$ (maximum likelihood reconstructions), with error bars obtained via standard Monte-Carlo simulations of quantum projection noise. Two ways to represent entanglement are chosen: Logarithmic negativity is an entanglement measure that can be computed for a generic bipartite mixed state $\rho$, from the trace norm of its partial transpose $\rho^{T_{A}}$ [34] $L N(\rho)=\log _{2}\left\|\rho^{T_{A}}\right\|_{1}$. This expression vanishes for unentangled states and we use it to quantify the degree of entanglement in the reduced 2-qubit density matrices $\rho^{(2)}$ of neighbouring spin pairs: $L N_{2}\left(\rho^{(2)}\right)=\log _{2}\left\|\rho^{T_{A}}\right\|_{1}=\log _{2}\left(2 \cdot\left|\sum_{n} \mu_{n}\right|+1\right)$, where $\mu_{n}$ are the negative eigenvalues of $\rho^{T_{A}}$. For a qualitative discussion of entanglement evolution in neighbouring spin triplets $\rho^{(3)}$ we define tripartite logarithmic negativity $L N_{3}$ as the geometric mean of the three bipartite logarithmic negativities (similar to the definition of tripartite negativity in [35]): $L N_{3}\left(\rho^{(3)}\right)=\sqrt[3]{L N_{2}\left(\rho_{I-J K}\right) \cdot L N_{2}\left(\rho_{J-I K}\right) \cdot L N_{2}\left(\rho_{K-I J}\right)}$, where $L N_{2}\left(\rho_{I-J K}\right)=\log _{2}\left(2 \cdot\left|\sum_{i} \tilde{\mu}_{i}\right|+1\right)$, with $\tilde{\mu}_{i}$ as negative eigenvalues of $\rho^{T_{I}}$, the partial transpose of $\rho^{(3)}$ with respect to subsystem $I$.

Light-like cones and interaction ranges. Figures $2 \mathrm{a}$ and $4 \mathrm{a}$ show light-like cones representing the maximum speed at which most of the quantum information and correlations spread in our system [23]. Since we do not have finite-range interactions, these are not strict maximum speeds, however they still provide a practically useful description of the spreading of correlations and information in our system [23]. The black lines $t=d / v$ in Figures $2 \mathrm{a}$ and $4 \mathrm{a}$ delineate the light-like cones for a version of our system with effective nearest-neighbour interactions only $\left(d=\left|i-i_{c}\right|\right.$ denotes the distance from the centre ion $i_{c}$ in terms of the ion sites $\left.i=1 \ldots N\right)$. The spread velocity $v$ is estimated as follows. We consider a nearest-neighbour only model of our system with homogenous coupling strength set to the average nearest-neighbour coupling of the original (full) coupling matrix $J_{i j}$. We calculate the eigenmode spectrum and determine the gradient between every pair of consecutive eigenvalues. The largest of these gradients corresponds to the maximum velocity $v_{\max }$ at which energy and correlations disperse in the truncated system. Finally we renormalise $v_{\max }$ by the algebraic tail of the original coupling matrix. Specifically, we choose the central ion $i_{c}=5$ (7) respectively for 8 (14) ions and average between the left and right algebraic tail, resulting in the normalisation factor $G=\frac{1}{2 J} \sum_{i \neq j}\left(J_{j, i_{c}}+J_{i_{c}, j}\right)$ such that $v=\frac{v_{\max }}{G}$ with averaged nearest-neighbour interaction $J$ (next section). To quantify the approximate interaction range in our system we find a best fit between the eigenmode spectrum of our full $J_{i j}$ system model with the eigenmode spectrum of an interaction that reduces with a power-law $J_{i j} \propto|i-j|^{-\alpha}$ 
[23]. The decay parameter $\alpha$ yielding the best fit gives an effective interaction range of $\alpha=1.58$ (8 spins) and $\alpha=1.27$ (14 spins).

Normalised time units $(\mathbf{1} / \mathbf{J})$. Time axes in our plots are labeled in two ways: one way indicates the real laboratory time (in ms) passed during the evolution (e.g. Figure 2a right y-axis), while the other way shows the time normalised by the averaged nearest-neighbour interaction strength of the original coupling matrix $J_{i j}$ (e.g. Figure 2a left y-axis): $J=\frac{1}{N-1} \sum_{i=1}^{N-1} J_{i, i+1}$.

Certified MPS tomography. Here we give an overview of how we identify an MPS estimate of the unknown state in the laboratory and determine a fidelity certificate with a statistical uncertainty. In the Supplementary Material Sec. IV A a more detailed description can be found and FIG. S1 shows a schematic overview. The experimental data comprises the outcomes of 1000 repetitions of the $3^{k}$ different measurement settings (each setting consists of a measurement basis for all $N$ spins with distinguishable $2^{N}$ outcomes). These samples are split into two parts of 500 samples each. The first part is used to obtain an MPS estimate $\left|\psi_{c}^{k}\right\rangle$ of the unknown state $\rho_{\text {lab }}$ in the laboratory. The second part is used to obtain a certificate, i.e., a lower bound on the fidelity between the unknown state $\rho_{\mathrm{lab}}$ and our MPS estimation $\left|\psi_{c}^{k}\right\rangle$ of this state. We split the data in order to ensure statistical independence of the process of obtaining the MPS estimate $\left|\psi_{c}^{k}\right\rangle$ and the process of obtaining the value of the fidelity lower bound.

The first part of the experimental data is used to estimate the $6^{k}$ local outcome probabilities on each of the $N-k+1$ blocks of $k$ neighbouring spins (see Measurements above). Linear inversion is used to obtain estimates of the local $k$-spin reduced density matrices. These reduced density matrices are input into the modified SVT algorithm from Ref. [2] to obtain a pure MPS estimate. This pure state is used as the start vector for the iterative likelihood maximization algorithm over pure states from Ref. [7]. Likelihood maximization uses the local outcome probabilities as input and returns an initial MPS estimate $\left|\psi_{\text {est }}\right\rangle$ of the unknown lab state. Both algorithms search for an MPS with a small bond dimension which is compatible with the local outcome probabilities. Usually, the initial estimate $\left|\psi_{\text {est }}\right\rangle$ reproduces the local outcome probabilities well but it may or may not be close to the unknown state $\rho_{\text {lab }}$ in the laboratory.

We certify the initial estimate $\left|\psi_{\text {est }}\right\rangle$ using a so-called parent Hamiltonian obtained from a set of candidate Hamiltonians (which may not correspond to any physical energy in the system). We compute the local reduced density matrices $\rho_{s}$ of $\left|\psi_{\text {est }}\right\rangle$ on spins $s, s+1, \ldots, s+k-1$. Candi- 
date Hamiltonians are given by $H_{\tau}=\sum_{s=1}^{N-k+1} \mathbb{1}_{1, \ldots, s-1} \otimes h_{s} \otimes \mathbb{1}_{s+k, \ldots, N}$ where $h_{s}=P_{\operatorname{ker}\left(T_{\tau}\left(\rho_{s}\right)\right)}$ is the orthogonal projection onto the kernel of the linear operator $T_{\tau}\left(\rho_{s}\right)$ and $T_{\tau}$ replaces eigenvalues of $\rho_{s}$ smaller than or equal to $\tau$ by zero. (If $\left|\psi_{\text {est }}\right\rangle$ is a so-called injective MPS [36] then it is the non-degenerate ground state of $H_{\tau=0}[36,37]$. This is generally not the case for our choice of $k$.) For all Hamiltonians $H_{\tau}$ we compute a ground state $\left|\psi_{\mathrm{GS}}\right\rangle$ and the smallest and second smallest eigenvalues $E_{0}$ and $E_{1}$. The typical range of $E_{1}$ is the interval $[0,1]$ (without units because $H_{\tau}$ is a sum of unit-less orthogonal projections). For 14 spins, we use an MPS-based ground state search [12]. Among all Hamiltonians $H_{\tau}$ with non-degenerate ground state and $E_{1}-E_{0}>10^{-6}$, we choose the Hamiltonian $H$ which minimizes

$$
c \mathcal{D}\left(\left|\psi_{\mathrm{est}}\right\rangle,\left|\psi_{\mathrm{GS}}\right\rangle\right)-\left(E_{1}-E_{0}\right)
$$

$c=5$ is a constant and $\mathcal{D}(|\psi\rangle,|\tilde{\psi}\rangle)=\||\psi\rangle\langle\psi|-| \tilde{\psi}\rangle\langle\tilde{\psi}| \|_{1} / 2=\sqrt{1-|\langle\psi \mid \tilde{\psi}\rangle|^{2}}$ is the trace distance [38]. We denote by $H$ the Hamiltonian that we have chosen and by $\left|\psi_{c}^{k}\right\rangle$ the non-degenerate ground state of $H ;\left|\psi_{c}^{k}\right\rangle$ is the final estimate of the unknown state in the laboratory $\rho_{\text {lab. }}$ (If all $H_{\tau}$ have degenerate ground states, we do not obtain a final estimate $\left|\psi_{c}^{k}\right\rangle$ or a fidelity lower bound.) Because $\left|\psi_{c}^{k}\right\rangle$ is a ground state of $H, H$ is called a parent Hamiltonian of $\left|\psi_{c}^{k}\right\rangle$. As the ground state $\left|\psi_{c}^{k}\right\rangle$ is non-degenerate, a lower bound to the fidelity between $\left|\psi_{c}^{k}\right\rangle$ and $\rho_{\text {lab }}$ is given by [2]

$$
\left\langle\psi_{c}^{k}\left|\rho_{\text {lab }}\right| \psi_{c}^{k}\right\rangle \geq 1-\frac{E-E_{0}}{E_{1}-E_{0}}=F_{c}^{k}
$$

where $E=\operatorname{tr}\left(H \rho_{\text {lab }}\right)$ is the energy of the unknown lab state in terms of $H$. Knowledge of local $k$-spin reduced density matrices enables us to determine $E$ because $H$ consists of terms $h_{s}$ which act only on $k$ neighbouring spins.

We use the second part of the experimental data to determine estimates of the fidelity lower bound $F_{c}^{k}$ and its statistical uncertainty caused by a finite number of measurements. In order to estimate the statistical uncertainty, we express the energy $E$ as weighted sum of local outcome probabilities, $E=\sum_{s=1}^{N-k+1} \sum_{i=1}^{6^{k}} c_{s i} p_{s i}$. The coefficients $c_{s i}$ are derived from the Moore-Penrose pseudoinverse of the linear map which takes a local reduced density matrix to local outcome probabilities. $E$ is estimated using estimates of the local outcome probabilities $p_{s i}$. We estimate the variance of our estimate of $E$ by estimating the covariances between each pair of local outcome probabilities. If two local outcome probabilities have been estimated from measurements in the same basis, their covariance is estimated from the measurement outcomes with a simple outcome counting scheme; otherwise, their covariance is equal to zero. Supplementary Material 
Sec. IV.A.4 provides full details and also considers estimating one outcome probability from more than one measurement basis is also considered.

Ideal model of MPS tomography. Main text Figure 3a shows fidelity lower bounds $F_{c}^{k}$ based on an ideal model of the tomographic process (Dashed lines). The ideal model takes the ideal time-evolved states $|\phi(t)\rangle=\exp \left(-i H_{\text {Ising }} t / \hbar\right)|\phi(0)\rangle$. From these states, the exact values of the $6^{k}$ probabilities describing the measurement outcomes of the $3^{k} k$-fold tensor products of the Pauli $\mathrm{X}, \mathrm{Y}$ and $\mathrm{Z}$ matrices for each of the $N-k+1$ local blocks are calculated. These probabilities are used to seed the MPS tomography algorithms. Computing the energy $E=\operatorname{tr}\left(H \rho_{\text {lab }}\right.$ ) of the (now known) ideal lab state $|\phi(t)\rangle$ in terms of the parent Hamiltonian $H$ is simplified considerably as we can compute the exact local reductions of $\rho_{\text {lab }}$. As a consequence, the resulting fidelity lower bounds (plotted in Figure 3a as dashed lines) are known without uncertainty. The same model is used to obtain the idealised fidelity lower bound for the 14 spin state after 4 ms of evolution (see below). Such a numerical simulation represents a highly idealised experiment, requiring perfect initial state preparation, simulator evolution, and an infinite number of perfect measurements that would be required to obtain exact knowledge of local reductions.

In experiments, 1000 measurements per setting were performed, leading to imperfect knowledge of local reductions. The outcome of a numerical simulation of this finite number of measurements (on ideal states) is described in the Supplementary Material Sec. IV.B and presented in FIG. S2. The results show that most of the differences between data and idealised model in Figure 3a are due to finite measurement number effects.

Errors in the initial simulator state. In the main text, we state that the differences between the experimentally-obtained and ideal-simulator model fidelity bounds $F_{c}^{3}$ at $t_{14}=4 \mathrm{~ms}$ are largely explained by errors in the initial Néel state preparation for 14 spins. The initial 14-spin Néel state was prepared with a (directly-measured) fidelity of $0.89 \pm 0.01$, compared to $0.967 \pm 0.006$ for the 8-spin case. This corresponds to a significantly larger error-per-particle for the 14-spin case (e.g. error per particle can be quantified by $\log (F) / N$, where $F$ is the state fidelity). In order to determine the effect of an error in the initial state on the MPS reconstruction, we repeat the numerical simulation of the ideal-simulator model with a noisy model initial state. Out of 1000 times that we prepared and measured the initial 14-spin Néel state in the Z-basis, 893 times we observed the Néel state, 93 times we observed a state with one spin flip error and 12 times we 
observed two spin flip errors. The noisy model initial state is built as an appropriately weighted mixture of the ideal Néel state and single spin flip errors. At $t_{14}=4 \mathrm{~ms}$, the fidelity lower bounds from the ideal model, the noisy model with 1000 simulated measurements and the experiment are $F_{c}^{3}=0.78,0.49 \pm 0.07$ and $0.39 \pm 0.08$. Of course, the described errors in the initial state are not the only errors in the experiment, however, we conclude that they are largely responsible for the difference between the fidelity lower bounds obtained from experimental data and from an idealised model of a perfect simulator.

MPS tomography is efficient for the dynamics of 1D local systems. Certified MPS tomography can fail because no parent Hamiltonian with non-degenerate ground state can be found. For a product state $|\psi(0)\rangle$ which evolves into $|\psi(t)\rangle=\exp (-i H t)|\psi(0)\rangle$ under a Hamiltonian $H$ with one-dimensional strictly finite-range interactions, we prove the following result in the Supplementary Material (Sec. VII): The state $|\psi(t)\rangle$ can be certified with measurement and post-processing resources which scale, at any fixed evolution time $t$, polynomially in $N$ and inverse-polynomially in $1-F_{\mathrm{LB}} . N$ is the number of spins and $F_{\mathrm{LB}}$ is the minimal permissible value of the fidelity lower bound. This means that there is a state $\left|\psi^{\prime}\right\rangle$ such that the fidelity between $|\psi(t)\rangle$ and $\left|\psi^{\prime}\right\rangle$ can be lower bounded by (at least) $F_{\mathrm{LB}}$ using only local measurements on $|\psi(t)\rangle$. In addition, the resources required to construct $\left|\psi^{\prime}\right\rangle$ obey the same polynomial scaling as it is obtained from the $\epsilon$-QCA decomposition shown in FIG. S11 [39]. Determining whether the certificate proof can be generalised to higher spatial dimensions will be the subject of future work.

Direct Fidelity Estimation. The fidelity lower bounds returned by the MPS certification procedure are lower bounds. That is, the actual overlap (fidelity) between the lab state $\rho_{\text {lab }}$ (generally mixed) and the output MPS $\left|\psi_{c}^{3}\right\rangle$ could take any value between the certificate and unity. We implement the method of direct fidelity estimation (DFE) [4, 5] in a 14 spin system, to estimate how big the overlap between the two states actually is.

The fidelity can be expressed as $F\left(\left|\psi_{c}^{3}\right\rangle, \rho_{\mathrm{lab}}\right)=\left\langle\psi_{c}^{3}\left|\rho_{\mathrm{lab}}\right| \psi_{c}^{3}\right\rangle=\sum_{k=1}^{4^{N}} \rho_{\mathrm{lab}}^{k} \sigma^{k}$ where $\rho_{\text {lab }}^{k}=\operatorname{tr}\left(P^{k} \rho_{\text {lab }}\right)$ is the lab state's expectation value and $\sigma^{k}=\left\langle\psi_{c}^{3}\left|P^{k}\right| \psi_{c}^{3}\right\rangle$ the expectation value of the MPS $\left|\psi_{c}^{3}\right\rangle$, with the normalised Pauli string operators $P^{k}$. The brute force approach of measuring all $4^{N}$ observables is impractical for systems composed of more than a few qubits as the number of observables to be measured increases with $N$. The DFE method leverages the knowledge of the MPS estimate to overcome this infeasibility: the expression above is replaced 
by a preferential summation over those values of $k$ for which MPS-estimate components $\sigma^{k}$ are likely to be large. In other words, more measurements are made in those Pauli operators $P^{k}$ for which $\left|\psi_{c}^{3}\right\rangle$ has a large expectation value. Therefore, we first rewrite the fidelity as the expectation value of the variable $\rho_{\mathrm{lab}}^{k} / \sigma^{k}$ over the probability distribution $q^{k} \stackrel{\text { def }}{=}\left(\sigma^{k}\right)^{2}$ : $F=\sum_{k=1}^{4^{N}} q^{k} \frac{\rho_{\text {lab }}^{k}}{\sigma^{k}}$. Next, we evaluate this using a Monte Carlo approach: We draw $M$ random indices $\left(k_{1}, k_{2}, \ldots k_{M}\right)$ with $k_{i} \in\left\{1,2, \ldots, 4^{N}\right\}$ according to the distribution $q^{k}$ and approximate the fidelity with $F \approx \bar{F}=\frac{1}{M} \sum_{i=1}^{M} \frac{\bar{\rho}_{\text {lab }}^{k_{i}}}{\sigma^{k_{i}}}$. In the experiment we set $M=250$ and repeat the measurements many times. Specifically, the number of copies $N_{k}$ spent to measure a particular Pauli operator $P^{k}$ is proportional to the inverse square of its calculated expectation value $\sigma^{k_{i}}$. This way we prevent the error in $\bar{F}$ to be dominated by those terms for which $\sigma^{k_{i}}$ is small. This sums to a total of $5 \times 10^{5}$ copies of the state. FIG. S10(a) shows the experimentally measured $\rho_{\text {lab }}^{k_{i}}$ and the corresponding calculated expectation values $\sigma^{k_{i}}$ for the $M=250$ different observables. FIG. S10(b) depicts the distribution of $\rho_{\text {lab }}^{k_{i}} / \sigma^{k_{i}}$ for different $i$, from which we infer the fidelity estimate and its error: $0.74 \pm 0.05$. For a more detailed discussion, in particular error analysis, see Supp. Mat. Sec. VI.

Resource cost for a constant estimation error in MPS tomography. If a quantum state of $N$ spins in a linear chain is the only state compatible with its local reductions on $k$ neighbouring spins, MPS tomography aims to reconstruct that state from precisely those local reductions. Reconstruction of a reduced density matrix on $k$ spins can be achieved by measuring in the $3^{k}$ bases described above. If we measure each basis on each of the $N-k+1$ local blocks $M$ times, the total number of measurements is $M_{T}=M(N-k+1) 3^{k}$. MPS tomography is efficient if it achieves a constant estimation error with a total number of measurements which scales polynomially with $N$. The estimation fidelity is given by $F=\left|\left\langle\psi_{\text {ideal }} \mid \psi_{\text {est }}\right\rangle\right|^{2}$ where $\left|\psi_{\text {ideal }}\right\rangle$ is the state we want to estimate and $\left|\psi_{\text {est }}\right\rangle$ is the estimate provided by MPS tomography. We quantify the estimation error with the trace distance $D=\sqrt{1-F}$ between the density matrices of the two pure states.

In order to determine whether MPS tomography is efficient, we carried out numerical simulations on a classical computer. Specifically, we take an $N$-spin state $\left|\psi_{\text {ideal }}\right\rangle$ that would be generated by a $3 \mathrm{~ms}$ quench of a nearest-neighbour model of our system with couplings and fields set to the average values of the 8-spin model. The half-chain von Neumann entropies of these states are around 0.58 (base-2 logarithm), i.e., the states contain significant amounts of entanglement. On these states, we simulate $M$ measurements in each $k$-spin basis as described in the last paragraph. Tomography was repeated ten times to account for fluctuations in the 
reconstruction error due to the finite number of measurements. Results of this simulation are shown in FIG. S3 for $N \in\{8,20,32,48,64\}$ spins. The left part of the figure shows that the estimation error decreases slightly with $N$ if the ratio $c=M / N^{2}$ is held constant. This means that an (at most) constant estimation error can be achieved with a total number of measurements given by $M_{T}=c N^{2}(N-k+1) 3^{k}$ which is only cubic in $N$. In principle, post-processing time must also increase at least with $N^{3}$ as this is how the size of the input data grows. However, with our implementation it takes only a short time to convert the input data into linearly many local expectation values. As a consequence, we observe that the time required by MPS tomography increases roughly with $\approx N^{1.2}$; in any case, post-processing time increases at most cubically with $N$. Hence, MPS tomography is efficient. Standard tomography is not efficient because the number of observables which have to be measured scales exponentially with $N$. Moreover, FIG. S3 right shows that the reconstruction error of MPS tomography scales roughly linearly with $1 / \sqrt{M}$ where $M$ is the number of measurements per basis.

The data that support the plots within this paper and other findings of this study are available from the corresponding author upon reasonable request.

[1] Vogel, K. \& Risken, H. Determination of quasiprobability distributions in terms of probability distributions for the rotated quadrature phase. Phys. Rev. A 40, 2847-2849 (1989).

[2] Cramer, M. et al. Efficient quantum state tomography. Nat. Commun. 1, 149 (2010).

[3] Gross, D., Liu, Y.-K., Flammia, S. T., Becker, S. \& Eisert, J. Quantum state tomography via compressed sensing. Phys. Rev. Lett. 105, 150401 (2010).

[4] Flammia, S. T. \& Liu, Y.-K. Direct fidelity estimation from few Pauli measurements. Phys. Rev. Lett. 106, 230501 (2011).

[5] da Silva, M. P., Landon-Cardinal, O. \& Poulin, D. Practical characterization of quantum devices without tomography. Phys. Rev. Lett. 107, 210404 (2011).

[6] Baumgratz, T., Gross, D., Cramer, M. \& Plenio, M. B. Scalable reconstruction of density matrices. Phys. Rev. Lett. 111, 020401 (2013).

[7] Baumgratz, T., Nüßeler, A., Cramer, M. \& Plenio, M. B. A scalable maximum likelihood method for quantum state tomography. New J. Phys. 15, 125004 (2013). 
[8] Tóth, G. et al. Permutationally invariant quantum tomography. Phys. Rev. Lett. 105, 250403 (2010).

[9] Cramer, M. et al. Spatial entanglement of bosons in optical lattices. Nature Commun. 4, 2161 (2013).

[10] Shabani, A. et al. Efficient measurement of quantum dynamics via compressive sensing. Phys. Rev. Lett. 106, 100401 (2011).

[11] Steffens, A. et al. Towards experimental quantum-field tomography with ultracold atoms. Nat. Commun. 6, 7663 (2015).

[12] Schollwöck, U. The density-matrix renormalization group in the age of matrix product states. Ann. Phys. 326, 96-192 (2011).

[13] Fannes, M., Nachtergaele, B. \& Werner, R. F. Finitely correlated states on quantum spin chains. Comm. Math. Phys. 144, 443-490 (1992).

[14] Hastings, M. B. Solving gapped Hamiltonians locally. Phys. Rev. B 73, 085115 (2006).

[15] Brandao, F. G. S. L. \& Horodecki, M. An area law for entanglement from exponential decay of correlations. Nat. Phys. 9, 721-726 (2013).

[16] Materials and methods are available as supplementary materials at the nature website.

[17] Lieb, E. \& Robinson, D. The finite group velocity of quantum spin systems. Commun. Math. Phys. 28, 251-257 (1972).

[18] Nachtergaele, B. \& Sims, R. Much ado about something: Why Lieb-Robinson bounds are useful. IAMP News Bulletin, 22-29 (October 2010).

[19] Cheneau, M. et al. Light-cone-like spreading of correlations in a quantum many-body system. Nature 481, 484-487 (2012).

[20] Brandão, F. G. S. L. \& Horodecki, M. Exponential decay of correlations implies area law. Commun. Math. Phys. 333, 761-798 (2015).

[21] Eisert, J. \& Osborne, T. J. General entanglement scaling laws from time evolution. Phys. Rev. Lett. 97, 150404 (2006).

[22] Bravyi, S., Hastings, M. B. \& Verstraete, F. Lieb-Robinson bounds and the generation of correlations and topological quantum order. Phys. Rev. Lett. 97, 050401 (2006).

[23] Jurcevic, P. et al. Quasiparticle engineering and entanglement propagation in a quantum many-body system. Nature 511, 202-205 (2014).

[24] Jurcevic, P. et al. Spectroscopy of interacting quasiparticles in trapped ions. Phys. Rev. Lett. 115, 100501 (2015).

[25] Hauke, P. \& Tagliacozzo, L. Spread of correlations in long-range interacting quantum systems. Phys. 
Rev. Lett. 111, 207202 (2013).

[26] Haffner, H. et al. Scalable multiparticle entanglement of trapped ions. Nature 438, 643-646 (2005).

[27] Schachenmayer, J., Lanyon, B. P., Roos, C. F. \& Daley, A. J. Entanglement growth in quench dynamics with variable range interactions. Phys. Rev. X 3, 031015 (2013).

[28] Richerme, P. et al. Non-local propagation of correlations in quantum systems with long-range interactions. Nature 511, 198-201 (2014).

[29] Kim, I. H. On the informational completeness of local observables. arXiv.org (2014). arXiv:1405.0137v1.

[30] Schindler, P. et al. A quantum information processor with trapped ions. New J. Phys. 15, 123012 (2013).

[31] Sidje, R. B. A software package for computing matrix exponentials. ACM Trans. Math. Softw. 24, 130-156 (1998).

[32] Jones, E., Oliphant, T., Peterson, P. et al. SciPy: Open source scientific tools for Python (2001). URL http://www.scipy.org/

[33] Ježek, M., Fiurášek, J. \& Hradil, Z. c. v. Quantum inference of states and processes. Phys. Rev. A 68, 012305 (2003).

[34] Plenio, M. B. Logarithmic negativity: A full entanglement monotone that is not convex. Phys. Rev. Lett. 95, 090503 (2005).

[35] Sabín, C. \& García-Alcaine, G. A classification of entanglement in three-qubit systems. EPJ D 48, 435-442 (2008).

[36] Perez-Garcia, D., Verstraete, F., Wolf, M. M. \& Cirac, J. I. Matrix product state representations. Quantum Inf. Comput. 7, 401 (2007).

[37] Baumgratz, T. Efficient system identification and characterization for quantum many-body systems. Ph.D. thesis, Ulm University (2014).

[38] Nielsen, M. A. \& Chuang, I. L. Quantum Computation and Quantum Information (Cambridge University Press, Cambridge, 2007), 9th edn.

[39] Osborne, T. J. Efficient approximation of the dynamics of one-dimensional quantum spin systems. Phys. Rev. Lett. 97, 157202 (2006).

[40] Monz, T. et al. 14-qubit entanglement: Creation and coherence. Phys. Rev. Lett. 106, 130506 (2011). 